\title{
An enhanced dietetic service to care homes improves effective management of residents at risk of malnutrition
}

\author{
C. Grantham, S. Whitelaw, T. Coulton and G. Walker \\ Leicestershire Nutrition and Dietetic Service, 11-12 Warren Park Way, Enderby, Leicester LE19 4SA, UK
}

The rising cost to GPs of prescribing oral nutritional supplements (ONS) has been identified locally as a high priority for action. In one district, there are 30 nursing and residential care homes providing care for 1026 residents. A 12 month pilot dietetic service was funded by the locality commissioning group to implement a care pathway to manage care home residents at risk of malnutrition in line with the recommendations of $\mathrm{NICE}^{(1)}$ and ensure appropriate prescribing. Care home staff used a local nutritional screening tool to identify a resident at high risk of malnutrition, the resident was referred to the dietitian who carried out an assessment and produced a care plan. If ONS were required, the dietitian confirmed this need to the patient's GP; if ONS were not appropriate they were discontinued. The patient was reviewed regularly by the dietitian against expected outcomes. Training was provided for care home staff to increase their knowledge and skills in relation to nutritional screening, monitoring and food first.

From September 2009 to August 2010, 133 patients were referred to the dietitian through this pathway.

- 60 patients $(45 \%)$ did not start ONS

- 28 patients $(21 \%)$ had ONS reduced or stopped

- 45 patients $(34 \%)$ started or continued to have ONS

In the second 6 months, there appeared to be improved nutritional screening, a better food first approach in the care homes and more appropriate dietetic referrals.

Following evaluation of the pilot, the service was funded for a further 5 months. The service was not fully reinstated until December 2010, meaning that there was a 3 month gap with no dietetic input. From December 2010 to March 2011, 79 referrals were received by the dietitian. 20 of these were for residents who had been seen the previous year and re-referred as their nutrition risk score had increased or they had significant weight loss during the period when there was no dietetic input.

- 35 patients $(44 \%)$ had ONS stopped or reduced

- 27 patients $(34 \%)$ did not start ONS, but advice was given on food first.

- 12 patients $(15 \%)$ had ONS started or increased

When supplements were stopped, the patient had a further dietetic review to ensure they maintained their nutritional status. Those patients with no ONS were then transferred back to the care home staff to monitor and refer again if their nutritional risk increased.

The results showed that, despite training and provision of tools to facilitate nutritional screening and care planning, continued dietetic input was needed to maintain ongoing effective management of these residents and sustain improvements. The barriers to effective ongoing implementation of the care pathway by care home staff when there is no regular input from a dietitian needs further investigation. The involvement of the dietitian led to more appropriate prescribing of ONS and produced significant savings to the GPs' prescribing budgets.

1. National Institute for Clinical Excellence (2006) Nutrition support in adults: oral nutrition support, enteral tube feeding and parenteral nutrition. Clinical guideline 32 . 